\title{
SECONDARY CHARACTERISTIC CLASSES FOR $k$-SPECIAL BUNDLES
}

\author{
BY \\ ROBERT E. MOSHER
}

1. Introduction. The secondary characteristic classes of a sphere bundle with vanishing Euler class were introduced by Massey [10]. These classes provide the additional information over and above the cohomology structure of the base needed to determine the mod 2 cohomology of the total space as ring, as module over the cohomology of the base, and as module over the mod 2 Steenrod algebra. It was for this purpose that these classes were originally defined. Subsequent work by Peterson and Stein [16], Meyer [13], and the author [14] led to many new properties of secondary characteristic classes, including analogues of the $\mathrm{Wu}$ and Whitney formulas and reinterpretation of these classes in terms of functional cohomology operations (yielding in turn simpler proofs of formulas of [16]).

In this paper we define secondary characteristic classes for an $n$-plane bundle $\xi$ such that the top $k$ Whitney classes $w_{n-k+1}, \ldots, w_{n}$ of $\xi$ vanish. For $k=1$, these classes are the classes of Massey. By a theorem of Massey and Peterson [12], these classes are the additional information needed to determine the cohomology of the total space of the associated bundle with fibre $V_{n, k}$. We then prove analogues of the $\mathrm{Wu}$ and Whitney formulas.

Our secondary classes will be defined in terms of a functional cohomology operation, involving relative cup-product, defined by the basic method of Steenrod [17] on "Thom" classes of the bundle. While our formal treatment of the algebraic properties of such operations, as well as the presentation of the $\mathrm{Wu}$ and Whitney formulas, has been greatly influenced by the paper of Meyer [13], the operations we consider have smaller indeterminacy and the results obtained from these operations have the sharpness comparable to [16]. For other approaches to functional operations, see [15], [3], and [13].

These secondary classes are different from those of Adem and Gitler [4], but are not entirely unrelated [9], [8].

The paper is organized as follows. In $\$ 2$ we state the main results on secondary characteristic classes. $\S \S 3$ and 4 contain the algebraic formalism of functional cohomology operations, while in $\S 5$ we list some topological examples. $\$ 6$ contains the definition and elementary properties of secondary characteristic classes; $\$ 7$ relates these classes to the problem of computing the cohomology of the total space

Presented in part to the Society, April 25, 1964 under the title Whitney formulas for secondary characteristic classes; received by the editors January 23, 1967. 
of an appropriate fibration. The Wu formula is proved in $\S 8$, and the Whitney formulas in $\$ 9$.

We would like to thank Professor F. P. Peterson, who originally suggested this problem to us, for many helpful conversations.

2. Results on secondary characteristic classes. In this section all cohomology is with $Z_{2}$ for coefficients.

Let $\xi$ be an $n$-plane bundle over $B$; let $k \leqq n$.

DEFINITION 2.1. $\xi$ is $k$-special if $w_{i}(\xi)=0, n-k<i \leqq n$.

For a $k$-special bundle $\xi$, we will define a family of secondary characteristic classes $\Phi(I ; j)(\xi) \in H^{q}(B)$ modulo a suitable indeterminacy, where $n-k<j \leqq n$, $I$ is a sequence $\left\{i_{1}, \ldots, i_{r}\right\}$ of nonnegative integers, and $q=j-1+\sum_{s} i_{s}$. These characteristic classes are the components of a multiple characteristic class $\Phi(\xi)$ which vanishes if $\xi$ admits $k$ linearly independent sections.

Let $E$ be the total space of the bundle associated to $\xi$ with fibre $V_{n, k}$, the Stiefel manifold of $k$-frames in $n$-space. Let $A$ be the mod 2 Steenrod algebra, and $R$ the semitensor product $H^{*}(B) \odot A$ [12]. In $\S 7$ we obtain the following consequence of [12, Theorem 5.2], which precisely describes $H^{*}(E)$, except for a certain extension.

THEOREM 2.2. Let $\xi$ be $k$-special. Then the R-algebra $H^{*}(E)$ is determined by $\Phi(\xi)$.

REMARK. Intuitively speaking, the $R$-algebra structure of $H^{*}(E)$ contains its ring structure, the action of $H^{*}(B)$, and the action of $A$. See [12] for the formal definitions.

In $\S 6$ we define certain polynomials $Q(I ; j s)$ in the Whitney classes $w_{1}, \ldots, w_{n-k}$. In $\S 8$ we prove the following analogue of the Wu formulas. (We omit to write the argument $\xi$ whenever clarity permits.)

THEOREM 2.3. Let $\xi$ be $k$-special. Then

$$
S q^{J} \Phi(I ; j)=\Phi(J, I ; j)+\sum_{s ; J^{\prime}+J^{\prime \prime}=J} S q^{J^{\prime}} Q(I ; j s) \cup \Phi\left(J^{\prime \prime} ; s\right)
$$

modulo the total indeterminacy.

Now suppose $\xi^{\prime}$ is an $n^{\prime}$-plane bundle over $B^{\prime}$. By the Whitney formula, if $\xi^{\prime}$ is $k$-special, so is the bundle $\xi \oplus \xi^{\prime}$ over $B \times B^{\prime}$. (We no longer assume $\xi k$-special.) In $\$ 9$ we prove the following Whitney formulas for secondary characteristic classes.

THEOREM 2.4. Let $\xi^{\prime}$ be $k$-special and assume $k \leqq n$. Then

$$
\Phi(I ; j)\left(\xi \oplus \xi^{\prime}\right)=\sum_{s+t=j ; I^{\prime}+I^{\prime \prime}=I} S q^{I^{\prime}} w_{s}(\xi) \otimes \Phi\left(I^{\prime \prime} ; t\right)\left(\xi^{\prime}\right)
$$

modulo the total indeterminacy.

If $B=B^{\prime}$, denote by $\xi+\xi^{\prime}$ the internal Whitney sum over $B$. 
THEOREM 2.5. Suppose $\xi^{\prime}$ is $k$-special, $k \leqq n$, and $B=B^{\prime}$. Then

$$
\Phi(I ; j)\left(\xi+\xi^{\prime}\right)=\sum_{I^{\prime}+I^{\prime \prime}=I_{i} s+t=j} S q^{I^{\prime}} w_{s}(\xi) \cup \Phi\left(I^{\prime \prime} ; t\right)\left(\xi^{\prime}\right)
$$

modulo the total indeterminacy.

3. Algebraic functional operations. In this section we recall the algebraic formalism of functional primary cohomology operations. We work in the category of locally finitely generated graded left $R$-modules over a graded ring $R$ and consider only homogeneous $R$-homomorphisms of appropriate degree.

Suppose $C$ is a chain complex of free $R$-modules. The portion $d_{1}: C_{1} \rightarrow C_{0}$ of $C$ determines a "functional transformation" as follows (writing $d$ for $d_{1}$ ). Let $\triangle$ be an exact triangle with vertices $M, L$, and $N$ and maps $\mu: M \rightarrow L, \lambda: L \rightarrow N$, and $\nu: N \rightarrow M$. Suppose $\varepsilon: C_{0} \rightarrow M$ is a map.

Definition 3.1. $\langle C, \varepsilon, \Delta\rangle$ is defined if $\varepsilon d: C_{1} \rightarrow M$ and $\mu \varepsilon: C_{0} \rightarrow L$ are the zero homomorphisms.

Definition 3.2. If $\langle C, \varepsilon, \Delta\rangle$ is defined, then $\langle C, \varepsilon, \Delta\rangle$ is the set of maps $\beta: C_{1} \rightarrow L$ such that $\lambda \beta=\alpha d$ for some $\alpha: C_{0} \rightarrow N$ satisfying $\nu \alpha=\varepsilon$.

Proposition 3.3. If $\langle C, \varepsilon, \triangle\rangle$ is defined, then $\langle C, \varepsilon, \triangle\rangle$ is nonempty and is a coset of $d^{\#} \operatorname{Hom}\left(C_{0}, L\right)+\mu_{\#} \operatorname{Hom}\left(C_{1}, M\right)$ in $\operatorname{Hom}\left(C_{1}, L\right)$.

The proof of 3.3 is standard.

We now elucidate some naturality properties of the symbol $\langle C, \varepsilon, \Delta\rangle$. First, naturality with respect to the triangle $\triangle$.

Proposition 3.4. Let $(m, l, n): \triangle^{\prime} \rightarrow \triangle$ be a map of exact triangles. Let $\varepsilon^{\prime}: C_{0} \rightarrow M^{\prime} ;$ put $\varepsilon=m \varepsilon^{\prime}: C_{0} \rightarrow M$. Suppose $\left\langle C, \varepsilon^{\prime}, \triangle^{\prime}\right\rangle$ is defined. Then $\langle C, \varepsilon, \triangle\rangle$ is defined and $l_{\#}\left\langle C, \varepsilon^{\prime}, \triangle^{\prime}\right\rangle \subset\langle C, \varepsilon, \Delta\rangle$.

Next we state a naturality property with respect to $C$, generalizing the most obvious statement to allow change of ring. Thus we assume we are given a ring homomorphism $g: R^{\prime} \rightarrow R$; via $g$, each $R$-module has a natural $R^{\prime}$-structure. However, we still assume that $C$ is $R$-free and that $C, \varepsilon$, and $\triangle$ are defined over $R$.

Proposition 3.5. Let $C^{\prime}$ be a free chain complex over $R^{\prime}$ and $f: C^{\prime} \rightarrow C$ an $R^{\prime}$-linear chain map. Let $\varepsilon: C_{0} \rightarrow M$; put $\varepsilon^{\prime}=\varepsilon f_{0}: C_{0}^{\prime} \rightarrow M$. Suppose $\langle C, \varepsilon, \triangle\rangle$ is defined. Then $\left\langle C^{\prime}, \varepsilon^{\prime}, \triangle\right\rangle$ is defined and $f_{1}^{\#}\langle C, \varepsilon, \Delta\rangle \subset\left\langle C^{\prime}, \varepsilon^{\prime}, \Delta\right\rangle$.

Propositions 3.4 and 3.5 follow by inspection from the appropriate diagrams, which we leave to the reader to draw.

For the next proposition we assume $C, \varepsilon$, and $\triangle$ are defined over $R ; C^{\prime}, \varepsilon^{\prime}$, and $\triangle^{\prime}$ defined over $R^{\prime}$; and $g: R^{\prime} \rightarrow R$.

Proposition 3.6. Let $f: C^{\prime} \rightarrow C$ and $(m, l, n): \triangle^{\prime} \rightarrow \triangle$ be $R^{\prime}$-linear maps such that $\varepsilon f_{0}=m \varepsilon^{\prime}$. Suppose $\langle C, \varepsilon, \triangle\rangle$ and $\left\langle C^{\prime}, \varepsilon^{\prime}, \triangle^{\prime}\right\rangle$ are defined. Then $f_{1}^{\#}\langle C, \varepsilon, \triangle\rangle$ $=l_{\#}\left\langle C^{\prime}, \varepsilon^{\prime}, \triangle^{\prime}\right\rangle$ in $\operatorname{Hom}\left(C_{1}^{\prime}, L\right)$ modulo $d^{\prime \#} \operatorname{Hom}\left(C_{0}^{\prime}, L\right)+\mu_{\#} \operatorname{Hom}\left(C_{1}^{\prime}, M\right)$. 
Proof. By 3.4 and 3.5 each side is contained in $\left\langle C^{\prime}, \varepsilon f_{0}, \Delta\right\rangle=\left\langle C^{\prime}, m \varepsilon^{\prime}, \Delta\right\rangle$, which has the asserted indeterminacy.

We now state the relation between algebraic functional operations and extensions, working over the fixed ring $R$. From the exact triangle $\triangle$ we may extract the short exact sequence

$$
S: 0 \rightarrow \operatorname{Coker} \mu \rightarrow N \rightarrow \operatorname{Ker} \mu \rightarrow 0
$$

and obtain the element $s \in \operatorname{Ext}_{R}^{1}(\operatorname{Ker} \mu, \operatorname{Coker} \mu)$ by applying the connecting homomorphism of $S$ to the identity map of $\operatorname{Ker} \mu$.

Suppose now that $\langle C, \varepsilon, \triangle\rangle$ is defined. Since $\operatorname{Im} \varepsilon \subset \operatorname{Ker} \mu$ and since $\varepsilon d=0$ we may view $\varepsilon: C \rightarrow \operatorname{Ker} \mu$ as an augmentation of the free complex $C$ to $\operatorname{Ker} \mu$.

Let $\pi: L \rightarrow$ Coker $\mu$ be the projection.

Proposition 3.7. $\pi_{\#}\langle C, \varepsilon, \triangle\rangle$ determines a unique element of $H^{1}(C$; Coker $\mu)$.

Proof. Let $\beta \in\langle C, \varepsilon, \triangle\rangle$. Then $d_{2}^{\#}(\pi \beta)=\lambda \beta d_{2}=\alpha d_{1} d_{2}=0$; hence every representative of $\pi_{\#}\langle C, \varepsilon, \triangle\rangle$ is a cocycle. Since $\pi \mu=0$, the indeterminacy of

$$
\pi_{\#}\langle C, \varepsilon, \triangle\rangle: C_{1} \rightarrow \operatorname{Coker} \mu
$$

is contained in Im $d_{1}^{\#}$, the group of coboundaries.

On the other hand, let $C^{\prime}$ be a projective resolution of $\operatorname{Ker} \mu$; let $f: C \rightarrow C^{\prime}$ cover the identity map of $\operatorname{Ker} \mu$. Consider

$$
f^{*}: \operatorname{Ext}_{R}^{1}(\operatorname{Ker} \mu, \operatorname{Coker} \mu)=H^{1}\left(C^{\prime} ; \operatorname{Coker} \mu\right) \rightarrow H^{1}(C ; \operatorname{Coker} \mu) .
$$

Proposition 3.8. $f^{*}(s)=\pi_{*}\langle C, \varepsilon, \triangle\rangle \in H^{1}(C$; Coker $\mu)$.

3.8 follows directly from the definitions of $\langle C, \varepsilon, \triangle\rangle$ and the connecting homomorphism of $S$.

4. Algebraic operations and relations. We now suppose that $C_{0}$ and $C_{1}$ have the fixed ordered homogeneous $R$-bases $\left\{c_{0 i}\right\}$ and $\left\{c_{1 j}\right\}$ respectively. Let $d$ be associated to the matrix $D=\left\{d_{j i}\right\}$ given by $d\left(c_{1 j}\right)=\sum_{i} d_{j i} c_{0 i}$. Let $\varepsilon$ be associated to the column vector $E=\left\{e_{i}\right\}$ given by $\varepsilon\left(c_{0 i}\right)=e_{i}$. Then to say $\langle C, \varepsilon, \triangle\rangle$ is defined is to say $D E=0$ and $\mu E=0$.

Of special interest is the value of $\langle C, \varepsilon, \Delta\rangle$ on the elements $c_{1 j}$, or, better still, on the vector $c_{1}=\left\{c_{1 j}\right\}$.

Definition 4.1. The set of vectors of form $\langle C, \varepsilon, \Delta\rangle\left(c_{1}\right)$ is denoted $\langle D, E, \Delta\rangle$. The operator $\langle D, \quad\rangle$ is called the functional operation associated with the matrix $D$.

Proposition 4.2. If $\langle D, E, \triangle\rangle$ is defined, then $\langle D, E, \triangle\rangle$ is nonempty. The indeterminacy of $\langle D, E, \triangle\rangle$ is the set of vectors of form $D l+\mu m$, where $l=\left\{l_{i}\right\}$ and $\boldsymbol{m}=\left\{\boldsymbol{m}_{j}\right\}$ are arbitrary vectors of $L$ and $M$ respectively.

4.2 is an immediate consequence of 3.3 . 
REMARK. In the usual language of functional operations and relations, we would say that $\langle D, E, \triangle\rangle$ is the value of the functional operation arising from the ordered set of relations $\sum_{i} d_{j i} e_{i}=0$, defined on the vector $E$ satisfying $\mu E=0$.

The naturality properties 3.4-3.6 yield formulas for the operations $\langle D, E, \triangle\rangle$. We state some of these.

Let $A=\left\{a_{k j}\right\}$ be a matrix over $R$.

Proposition 4.3. Suppose $\langle D, E, \triangle\rangle$ is defined. Then $A\langle D, E, \triangle\rangle \subset\langle A D, E, \triangle\rangle$.

Proof. Construct a diagram

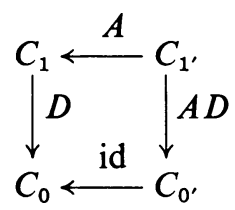

and apply 3.5 .

Proposition 4.4. Suppose $\langle D, E, \triangle\rangle$ and $\left\langle D^{\prime}, E^{\prime}, \triangle\right\rangle$ are defined. Then $\left\langle D+D^{\prime}, E, \triangle\right\rangle \subset\langle D, E, \triangle\rangle+\left\langle D^{\prime}, E, \triangle\right\rangle$.

Proof. Apply 3.5 twice in the following diagram, keeping track of the indeterminacy.

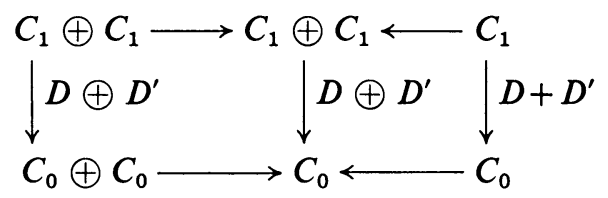

Proposition 4.5. Suppose $\langle A D, E, \triangle\rangle$ is defined. Then

$$
\langle A D, E, \triangle\rangle \subset\langle A, D E, \triangle\rangle \text {. }
$$

The proof is similar to that of 4.3 .

REMARK. It is perhaps apparent now why we have introduced the complex $C$ rather than, as in [13], simply the free module $C_{0}$ and a homogeneous element of $\operatorname{Ker} \varepsilon$. Firstly, we wish to be able to consider vector-valued operations with the "diagonal" indeterminacy rather than the "total" indeterminacy, the sum of the indeterminacies of the component operations. Secondly, the linearity and changeof-variables properties 4.3-4.5 are more transparent. Thirdly, the formalism of the. relation to extensions occurs more naturally.

5. Topological examples. In algebraic topology the usual source of exact triangles with rich algebraic structure is the cohomology sequence of a pair (or of a fibration in the stable range); pairs arise from maps. Thus for a map $f: Y \rightarrow X$, assumed an inclusion, we take for the exact triangle $\triangle$ the cohomology sequence of $f$, for some suitable cohomology theory and ring $R$, and leave open for the moment the question of which vertex will play the preferred rôle of $M$.

In particular, for a multiplicative theory, we may take $R=H^{*}(X)$, where $R$ acts 
on $H^{*}(Y)$ via $f^{*}$ and on $H^{*}(X, Y)$ via relative cup-product, since (under suitable sign conventions) $\triangle$ is a triangle of $R$-maps, according to the coboundary formula. For cohomology with coefficients in the prime field $Z_{p}$ we may take $R=A$, the mod $p$ Steenrod algebra. Or we may combine these two, taking $R=H^{*}(X) \odot A$, the semitensor product.

In these examples if we take $M=H^{*}(X)$ we obtain operations of the type considered in [13]. However, we note the possibility of taking $M=H^{*}(X, Y)$ to yield a functional operation involving relative cup-product. Our formulation of secondary characteristic classes is in exactly such terms.

6. $k$-special bundles. In this section we define the secondary characteristic classes of $k$-special vector bundles and prove their elementary properties. For the remainder of the paper all cohomology is with $Z_{2}$ as coefficients.

To fix notations, let $\xi$ be an $n$-plane bundle over $B$; let $k \leqq n$. Let $p: E \rightarrow B$ be the associated bundle with fibre $V_{n, k}$. Let $T$ be the Thom space (or cofibre) of $p$-i.e., $T=Z / E$, where $Z$ is the mapping cylinder of $p$. $T$ has a natural base-point, and $H^{*}(T)$ denotes reduced cohomology. Let $j: B \rightarrow T$ be the inclusion.

Following Massey and Peterson [12], we recall that the universal example for such an associated bundle is given by $E=B O(n-k)$ and $B=B O(n)$. In this case $j^{*}: H^{*}(T) \rightarrow H^{*}(B)$ maps $H^{*}(T)$ monomorphically onto the ideal $\mathscr{I}=\mathscr{I}(n, k)$ generated by the $w_{i}, n-k<i \leqq n$, in the polynomial ring $Z_{2}\left[w_{1}, \ldots, w_{n}\right]$.

Proposition 6.1. I is closed under the action of the Steenrod algebra A.

DefinItION 6.2. For $n-k<i, j \leqq n$, let $Q(I ; j i)$ be the unique polynomial in $w_{1}, \ldots, w_{n-k}$ such that

$$
S q^{I} w_{j}=\sum_{i} Q(I ; j i) \cup w_{i} \text { modulo } \mathscr{I}^{2}
$$

Strictly speaking, we should write $Q(I ; j i)_{n, k}$. In view of the next proposition, we usually do not.

Proposition 6.3. Let $n-k<i, j \leqq n$. Then

(a) $Q(I ; j i)=0$ if $i<j$.

(b) $Q(I ; j i)_{n+1, k+1}=Q(I ; j i)_{n, k}$.

(c) $Q(I ; j i)_{n, k+1}=Q(I ; j i)_{n, k}\left(w_{1}, \ldots, 0\right)$.

6.1 is obvious, as is the proof of 6.3.

REMARK. The $Q(I ; j i)$ may be computed from the $\mathrm{Wu}$ formulas.

Definition 6.4. For $n-k<i \leqq n$, let $U_{i} \in H^{i}(T)$ be the unique class satisfying $j^{*}\left(U_{i}\right)=w_{i}$.

By naturality $U_{i}(\xi)$ is defined for arbitrary $\xi$ and satisfies $j^{*}\left(U_{i}(\xi)\right)=w_{i}(\xi)$. The classes $U_{i}(\xi)$ are called the Thom classes of $\xi$.

Definition 6.5. Let $M(\xi)$ be the $H^{*}(B)$-submodule of $H^{*}(T)$ generated by the $U_{i}$, where $H^{*}(B)$ acts on $H^{*}(T)$ by relative cup-product. 
Proposition 6.6. $S q^{I} U_{j}(\xi)=\sum_{i} Q(I ; j i)(\xi) \cup U_{i}(\xi) \bmod M(\xi)^{2}$.

Proof. In the universal example, since $j^{*}$ is a monomorphism, 6.6 follows from 6.2. The general case follows by naturality.

We now consider $k$-special bundles, as defined in 2.1.

Proposition 6.7. Suppose $\xi$ is $k$-special. Then

(a) $j^{*}=0$; in particular, $j^{*}\left(U_{i}\right)=0$.

(b) $M^{2}=0$.

(c) $S q^{\prime} U_{j}=\sum_{i} Q(I ; j i) \cup U_{i}$.

(d) $M$ is free as an $H^{*}(B)$-module with the $U_{1}$ as $H^{*}(B)$-basis and is closed under the action of $A$.

Proof. (a) is well known. $U_{i} \cup U_{j}=w_{i} \cup U_{j}=0$, which proves (b), and thus, by 6.6, (c). See (c) and [12] for (d).

We now have verified all the conditions needed to define secondary characteristic classes for the $k$-special bundle $\xi$. Put $R=H^{*}(B) \odot A$ and let $M=H^{*}(T)$, $L=H^{*}(B)$, and $N=H^{*}(E)$ be the vertices of the exact triangle $\triangle$. Let $U$ be the vector $\left\{U_{i}\right\}$ over $H^{*}(T)$ and $D(I ; j)$ the row matrix over $R$ defined by $D(I ; j i)$ $=Q(I ; j i)+\delta_{j i} S q^{I}$, where $\delta_{j i}$ is the Kronecker symbol. In matrix language, $D(I ; j)$ is a row of the matrix $D(I)=Q(I)+\delta S q^{I}$.

Definition 6.8. Let $\xi$ be $k$-special. Then the secondary characteristic class $\Phi(I ; j)(\xi)$ is defined by $\Phi(I ; j)=\langle D(I ; j), U, \Delta\rangle$.

Thus $\Phi(I ; j)$ is associated with the relation 6.7(c).

Proposition 6.9. $\Phi(I ; j)(\xi)$ is a homogeneous coset in $H^{q}(B)$ and has indeterminacy $D(I ; j) b$, where $q=j-1+\operatorname{deg} I$ and $b=\left\{b_{i}\right\}$ is an arbitrary vector of $H^{*}(B)$.

Proposition 6.10. Let $g: \xi \rightarrow \xi^{\prime}$ be a map of $k$-special n-plane bundles. Then $g^{*} \Phi(I ; j)\left(\xi^{\prime}\right) \subset \Phi(I ; j)(\xi)$.

Proofs. 6.9 is obvious. 6.10 is an easy application of 3.6, involving a change of rings induced by $g^{*}: H^{*}\left(B^{\prime}\right) \rightarrow H^{*}(B)$.

The next proposition asserts that $\Phi(I ; j)(\xi)$ is independent of the choice of $k$ such that $\Phi(I ; j)$ is defined.

Proposition 6.11. Suppose $\Phi$ is $(k+1)$-special and $n-k<j \leqq n$. Then $\Phi(I ; j)_{k}(\xi)$ $=\Phi(I ; j)_{k+1}(\xi)$.

Proof. The projection $V_{n, k+1} \rightarrow V_{n, k}$ induces a map of associated bundles, which in turn induces an $R$-map of the relevant triangles. By 6.3(a) and 6.3(c), 3.4 applies and asserts that $\Phi(I ; j)_{k} \subset \Phi(I ; j)_{k+1}$. But by 6.3 again, the indeterminacies coincide and the inclusion is an equality.

Now suppose $\Phi$ is $k$-special and $\Phi(I ; j)(\xi)$ is defined. Let $\xi+1$ be the bundle over $B$ obtained by adding a trivial bundle. Then $\xi+1$ is $(k+1)$-special; thus $\Phi(I ; j)(\xi+1)$ is defined. 
Proposition 6.12. $\Phi(I ; j)(\xi) \subset \Phi(I ; j)(\xi+1)$.

The proof of 6.12 is similar to that of 6.11 but makes use of the inclusion $V_{n, k} \rightarrow V_{n+1, k+1}$ and of 6.3(b). The asserted inclusion is not in general an equality, for the term $Q(I ; j i)$ with $i=n+1$ in $D(I ; j)$ increases the indeterminacy, as in 7.8.

7. Secondary characteristic classes and $H^{*}(E)$. In this section we prove 2.2, relating secondary characteristic classes to the determination of $H^{*}(E)$.

Let $\xi$ be a $k$-special $n$-plane bundle. By 6.7 the exact triangle $\triangle$ is simply the short exact sequence $\triangle(\xi): 0 \rightarrow H^{*}(B) \rightarrow H^{*}(E) \stackrel{\delta}{\rightarrow} H^{*}(T) \rightarrow 0$. In $H^{*}(T)$ we have the submodule $M$; following [12], put $N(\xi)=\delta^{-1} M(\xi)$, yielding the exact triangle (in fact a short exact sequence)

$$
S(\xi): 0 \rightarrow H^{*}(B) \rightarrow N \rightarrow M \rightarrow 0 .
$$

By $6.7 S(\xi)$ is a short exact sequence of $R$-modules.

THEOREM 7.1 [12]. Let $\xi$ be $k$-special. Then the R-algebra $H^{*}(E)$ is determined by the R-algebra $H^{*}(B)$ and the $R$-module $N$.

It remains to determine $N$.

By 3.4 it is apparent that the secondary characteristic classes of $\xi$ may be defined in the sequence $S(\xi)$ with no change in indeterminacy.

Definition 7.2. Let $\Phi(\xi)$ be the secondary characteristic class associated with the doubly indexed family of relations

$$
S q^{I} U_{j}=\sum_{i} Q(I ; j i) \cup U_{i}
$$

indexed by $j$ and the set of sequences $I$.

REMARK. The $I ; j$ th component of $\Phi$ is simply $\Phi(I ; j)$, but $\Phi$ has the "diagonal" indeterminacy. Precisely, 4.3 shows that if $z=\{z(I ; j)\} \in \Phi$, then $z(I ; j) \in \Phi(I ; j)$, but not conversely.

Now let $d: C_{1} \rightarrow C_{0}$ and $\varepsilon: C_{0} \rightarrow M$ be associated with 7.3.

Proposition 7.4. The sequence $C_{1} \rightarrow C_{0} \rightarrow M \rightarrow 0$ is exact.

Proof. Exactness at $M$ is obvious. Exactness at $C_{0}$ follows from the freeness of $M$ over $H^{*}(B)$ and the observation that the $S q^{I}$ generate $A$.

REMARK. It would thus be possible, for the succeeding discussion, to replace in 7.3 the set of sequences $I$ with any set of generators for the algebra $A$.

By 7.4 we may extend the augmented complex $d: C_{1} \rightarrow C_{0}$ to a free resolution $C$ of $M$. By $3.7 \Phi$ determines an element, also denoted $\Phi$, of $\operatorname{Ext}_{R}^{1.1}\left(M, H^{*}(B)\right)$. Recall that the sequence $S$ also determines an element $s$ of this group.

Proposition 7.5. $\Phi(\xi)=s(\xi) \in \operatorname{Ext}_{R}^{1.1}\left(M, H^{*}(B)\right)$.

Proof. Immediate from 3.8.

Proof of 2.2 is immediate from 7.1 and 7.5. 
Proposition 7.6. If $\xi$ admits $k$ linearly independent sections, then $\Phi(\xi)=0$.

Proof. $\xi$ admits $k$ linearly independent sections if and only if the bundle $p: E \rightarrow B$ admits a section, in which case the sequence $S(\xi)$ splits over $R$.

EXAMPLE 7.7. Let $n+1=2^{m}(2 s+1)$. Then the theorem of Steenrod and J. H. C. Whitehead [19] asserts that the tangent bundle $\tau$ of $S^{n}$ does not admit a $2^{m}$-field. Their proof for $m$ and $s$ positive may be reinterpreted as follows. Consider $\tau$ as a $k$-special $n$-plane bundle with $k=2^{m}$. Then $\Phi(k ; n-k+1)(\tau) \neq 0$.

EXAMPLE 7.8. $\tau+1$ is trivial; thus by $7.6 \Phi(k ; n-k+1)(\tau+1)=0$, which apparently contradicts 6.12 . It does not. While the indeterminacy of $\Phi(k ; n-k+1)(\tau)$ is zero, that of $\Phi(k ; n-k+1)(\tau+1)$ is the entire group $H^{n}\left(S^{n}\right)$.

8. Proof of Theorem 2.3. In this section we prove the $\mathrm{Wu}$ formulas for secondary characteristic classes. At this point we caution the reader that the symbol $S q^{I} b$, for $b \in H^{*}(B)$, has its usual meaning, but $S q^{I} \cdot b$ will denote the product $\left(1 \odot S q^{I}\right)(b \odot 1)$ in $R$ and has value $\sum_{I^{\prime}+I^{\prime \prime}=I} S q^{I^{\prime}} b \odot S q^{I^{\prime \prime}}$; in general the dot will indicate product in $R$. We use matrix notation where possible-for example, $S q^{J} Q(I)$ is the matrix having $S q^{J} Q(I ; j i)$ as $j i$ th entry, while the cup-product in the following lemma is matrix multiplication, under cup-product, in $H^{*}(B)$.

LEMMA 8.1. Let $\xi$ be $k$-special. Then

$$
Q(J, I)=\sum_{J^{\prime}+J^{\prime \prime}=J} S q^{J^{\prime}} Q(I) \cup Q\left(J^{\prime \prime}\right) .
$$

The proof is entirely analogous to that of 12.3 of [13], taking into account 6.7.

Lemma 8.2. Let $\xi$ be $k$-special. Then

$$
S q^{J} \cdot D(I)=D(J, I)+\sum_{J^{\prime}+J^{\prime \prime}=J}\left\{S q^{J^{\prime}} Q(I)\right\} \cdot D\left(J^{\prime \prime}\right) .
$$

Proof. Routine computation, using 8.1.

Proof of Theorem 2.3. Recall that $\Phi(I ; j)$ is $\langle D(I ; j), U, \triangle\rangle$, which we abbreviate as $\langle D(I ; j)\rangle$ here. Modulo appropriate indeterminacies, repeated applications of 4.3 and 4.4 , together with 8.2 , yield

$$
\begin{aligned}
S q^{J} \Phi(I ; j) & =S q^{J}\langle D(I ; j)\rangle=\left\langle S q^{J} D(I ; j)\right\rangle \\
& =\left\langle D(J, I ; j)+\sum_{J^{\prime}+J^{\prime \prime}=J}\left\{S q^{J^{\prime}} Q(I ; j)\right\} \cdot D\left(J^{\prime \prime}\right)\right\rangle \\
& =\langle D(J, I ; j)\rangle+\sum_{J^{\prime}+J^{\prime \prime}=J ; s} S q^{J^{\prime}} Q(I ; j s) \cup\left\langle D\left(J^{\prime \prime} ; s\right)\right\rangle \\
& =\Phi(J, I ; j)+\sum_{J^{\prime}+J^{\prime \prime}=J ; s} S q^{J^{\prime}} Q(I ; j s) \cup \Phi\left(J^{\prime \prime} ; s\right) .
\end{aligned}
$$

A computation verifies that the equality holds modulo the sum of the indeterminacies of the various terms of 2.3-i.e., modulo the total indeterminacy. This completes the proof of 2.3 .

We state the following sharpening of 2.3 and leave the proof to the reader. 
THEOREM 8.3. Let $\xi$ be $k$-special. Let $z \in \Phi(\xi)$. Then

$$
S q^{J} z(I ; j)=z(J, I ; j)+\sum_{J^{\prime}+J^{\prime \prime}=J ; s} S q^{J^{\prime}} Q(I ; j s) \cup z\left(J^{\prime \prime} ; s\right) .
$$

9. Whitney sums and the proof of Theorems 2.4 and 2.5. Let $\xi, \xi^{\prime}$ be $n, n^{\prime}$-plane bundles over $B, B^{\prime}$ respectively. Fix $k \leqq n, n^{\prime}$. Then the external generalized Whitney sum $p \oplus p^{\prime}: E \oplus E^{\prime} \rightarrow B \times B^{\prime}$ is defined as follows.

Let $p \times 1: E \times B^{\prime} \rightarrow B \times B^{\prime}$ and $1 \times p^{\prime}: B \times E^{\prime} \rightarrow B \times B^{\prime}$ be the bundles induced over $B \times B^{\prime}$ from $p$ and $p^{\prime}$ by left and right projections respectively. Then $p \oplus p^{\prime}$ is taken as the (internal) generalized Whitney sum $(p \times 1)+\left(1 \times p^{\prime}\right)$ defined in [5]; $p \oplus p^{\prime}$ is a bundle with fibre $V_{n, k}{ }^{*} V_{n^{\prime}, k}$ and group $O(n) \times O\left(n^{\prime}\right)$.

A natural homeomorphism of pairs $g: Z\left(p \oplus p^{\prime}\right), E \oplus E^{\prime} \rightarrow Z \times Z^{\prime}, E \times Z^{\prime}$ $\cup Z \times E^{\prime}$, extending the identity of $B \times B^{\prime}$, is given by

$$
g\left\{t\left(e, p^{\prime}\left(e^{\prime}\right)\right),(1-t)\left(p(e), e^{\prime}\right), u\right\}=\left\{(e, v),\left(e^{\prime}, w\right)\right\},
$$

where $w=u$ and $v=1+2 t(u-1)$ for $0 \leqq t \leqq \frac{1}{2}$, while $w=2(1-u) t-1+2 u$ and $v=u$ for $\frac{1}{2} \leqq t \leqq 1$. Since $T=Z / E, g$ induces a natural homeomorphism from the Thom space $T\left(p \oplus p^{\prime}\right)$ to $T(p) \wedge T\left(p^{\prime}\right)$ extending the identity of $B \times B^{\prime}$.

Let $\xi^{\prime \prime}=\xi \oplus \xi^{\prime}$ be the Whitney sum $n^{\prime \prime}\left(=n+n^{\prime}\right)$-plane bundle over $B^{\prime \prime}=B \times B^{\prime}$,

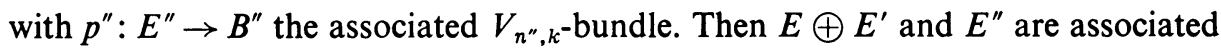
to the same principal $O(n) \times O\left(n^{\prime}\right)$-bundle.

Let $f: V_{n, k}{ }^{*} V_{n^{\prime}, k} \rightarrow V_{n^{\prime \prime}, k}$ be the intrinsic join of James [6]. Since $f$ is $O(n) \times O\left(n^{\prime}\right)$ equivariant, $f$ induces a natural bundle map $f: E \oplus E^{\prime} \rightarrow E^{\prime \prime}$ and a natural map $f: T \wedge T^{\prime} \rightarrow T^{\prime \prime}$, natural with respect to pairs $\xi$, $\xi^{\prime}$ of $n, n^{\prime}$-plane bundles. We now describe the behavior of the Thom classes under $f$.

Proposition 9.1. $f^{*}\left(U_{i}^{\prime \prime}\right)=\sum_{s+t=i} U_{s} \otimes U_{t}^{\prime}$.

Proof. It suffices to verify 9.1 in the universal example $B=B O(n)$ and $B^{\prime}$ $=B O\left(n^{\prime}\right)$, where 9.1 is an immediate consequence of the Whitney formula.

COROLlARY 9.2. Let $h_{i} \in H^{i-1}\left(V_{n, k}\right), n-k<i \leqq n$, be the members of the standard simple system of generators. Then under the intrinsic join $f$,

$$
f^{*}\left(h_{i}^{\prime \prime}\right)=\sum_{s+t=i} h_{s} * h_{t}^{\prime}
$$

Proof. Apply 9.1 with $B$ and $B^{\prime}$ each a point.

We now turn attention to the case $\xi^{\prime}$, and hence $\xi^{\prime \prime}, k$-special.

Let $A$ be the matrix $A(i t)=w_{i-t}$.

Lemma 9.3. Let $\xi^{\prime}$ be $k$-special. Then

$$
Q^{\prime \prime}(I) \cup A=\sum_{I^{\prime}+I^{\prime \prime}=I} S q^{I^{\prime}} A \cup Q^{\prime}\left(I^{\prime \prime}\right) .
$$

Proof. The proof is formally similar to that of (12.5) of [13], but more delicate, since 6.7 is not valid for arbitrary bundles. The calculation is made in an appropriate universal example; namely, take $B=B O(n)$ and take for $B^{\prime}$ the base space of 
a universal example for $k$-special $n^{\prime}$-plane bundles (see [11] for existence). Under the cohomology map induced by $B^{\prime} \rightarrow B O\left(n^{\prime}\right)$, the polynomial subalgebra of $H^{*}\left(B O\left(n^{\prime}\right)\right)$ generated by the $w_{i}^{\prime}$ for $i \leqq n^{\prime}-k$ is monomorphically embedded in $H^{*}\left(B^{\prime}\right)$. We have the map $f: T \wedge T^{\prime} \rightarrow T^{\prime \prime}$ and the monomorphism

$$
j^{*} \otimes 1: H^{*}(T) \otimes H^{*}\left(T^{\prime}\right) \rightarrow H^{*}(B) \otimes H^{*}\left(T^{\prime}\right) .
$$

The $U_{i}^{\prime}$ are independent over $H^{*}(B) \otimes H^{*}\left(B^{\prime}\right)$ in $H^{*}(B) \otimes H^{*}\left(T^{\prime}\right)$. Thus we may equate coefficients in the expansions of $\left(j^{*} \otimes 1\right) f^{*}\left(S q^{I} U_{i}^{\prime \prime}\right)$ and $\left(j^{*} \otimes 1\right) S q^{I} f^{*}\left(U_{i}^{\prime \prime}\right)$ expanding by $6.7(\mathrm{c})$ and 9.1 . So doing completes the proof.

LEMMA 9.4. Let $\xi^{\prime}$ be $k$-special. Then

$$
D^{\prime \prime}(I) \cdot A=\sum_{I^{\prime}+I^{\prime \prime}=I} S q^{I^{\prime}} A \cdot D^{\prime}\left(I^{\prime \prime}\right) .
$$

Proof. Compute, using 9.3.

Proof of Theorem 2.4. Let $h: Z \times E^{\prime} \rightarrow E \oplus E^{\prime}=E \times Z^{\prime} \cup Z \times E^{\prime}$ be the inclusion, and, as usual, let $f$ be induced by the intrinsic join. We identify $H^{*}(B)$ with $H^{*}(Z)$. Consider the diagram

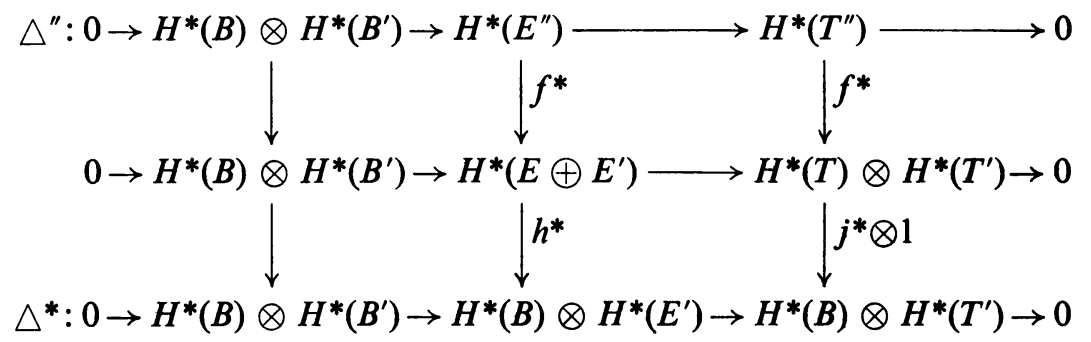

and the sequence

$$
\triangle^{\prime}: 0 \rightarrow H^{*}\left(B^{\prime}\right) \rightarrow H^{*}\left(E^{\prime}\right) \rightarrow H^{*}\left(T^{\prime}\right) \rightarrow 0 .
$$

By 6.4 and $9.1,\left(j^{*} \otimes 1\right) f^{*}\left(U^{\prime \prime}\right)=A U^{\prime}$. Thus application of the various naturality formulas, together with 9.4 , yields the following equalities, modulo appropriate indeterminacies.

$$
\begin{aligned}
\Phi(I ; j)\left(\xi \oplus \xi^{\prime}\right) & =\left\langle D^{\prime \prime}(I ; j), U^{\prime \prime}, \triangle^{\prime \prime}\right\rangle \\
& =\left\langle D^{\prime \prime}(I ; j), A U^{\prime}, \triangle^{*}\right\rangle \\
& =\left\langle D^{\prime \prime}(I ; j) \cdot A, U^{\prime}, \triangle^{*}\right\rangle \\
& =\left\langle\sum_{I^{\prime}+I^{\prime \prime}=I} S q^{I^{\prime}} A(j) \cdot D^{\prime}\left(I^{\prime \prime}\right), U^{\prime}, \Delta^{*}\right\rangle \\
& =\sum_{I^{\prime}+I^{\prime \prime}=I} S q^{I^{\prime}} A(j) \otimes\left\langle D^{\prime}\left(I^{\prime \prime}\right), U^{\prime}, \triangle^{\prime}\right\rangle \\
& =\sum_{I^{\prime}+I^{\prime \prime}=I: t} S q^{I^{\prime}} A(j t) \otimes\left\langle D^{\prime}\left(I^{\prime \prime} ; t\right), U^{\prime}, \triangle^{\prime}\right\rangle \\
& =\sum_{I^{\prime}+I^{\prime \prime}=I ; t} S q^{I^{\prime}} w_{j-t}(\xi) \otimes \Phi\left(I^{\prime \prime} ; t\right)\left(\xi^{\prime}\right)
\end{aligned}
$$


as asserted. We omit the routine verification that equality holds modulo the sum of the indeterminacies of each side of 2.4. This verification completes the proof of 2.4. 2.5 is a direct consequence of 2.4 and 6.10 . We now state the analogue of 8.3.

TheOREM 9.5. Suppose $\xi^{\prime}$ is $k$-special and $k \leqq n$. Let $z \in \Phi\left(\xi^{\prime}\right)$. Put

$$
y(I ; j)=\sum_{I^{\prime}+I^{\prime \prime}=I ; s+t=j} S q^{I^{\prime}} w_{s} \otimes z\left(I^{\prime \prime} ; t\right) .
$$

Then $\boldsymbol{y} \in \Phi\left(\xi \oplus \xi^{\prime}\right)$.

Corollary 9.6. Suppose $\xi$ and $\xi^{\prime}$ are both $k$-special. Then $\Phi\left(\xi \oplus \xi^{\prime}\right)=0$.

\section{REFERENCES}

1. J. F. Adams, On the structure and applications of the Steenrod algebra, Comment. Math. Helv. 32 (1958), 180-214.

2. - - On the nonexistence of elements of Hopf invariant one, Ann. of Math. (2) 72 (1960), 20-104.

3. J. Adem, Sobre operaciones cohomologiques secundarias, Bol. Soc. Mat. Mexicana 7 (1962), 95-110.

4. J. Adem and S. Gitler, Secondary characteristic classes and the immersion problem, Bol. Soc. Mat. Mexicana (1964), 53-78.

5. I. M. Hall, The generalized Whitney sum, Quart. J. Math. Oxford (2) 16 (1965), 360-384.

6. I. M. James, The intrinsic join: A study of the homotopy groups of Stiefel manifolds, Proc. London Math. Soc. 8 (1958), 507-535.

7. S. MacLane, Homology, Springer-Verlag, Berlin, 1963.

8. M. Mahowald, Some Whitehead products in $S^{n}$, Topology 4 (1965), 17-26.

9. M. Mahowald and F. P. Peterson, Secondary cohomology operations on the Thom class, Topology 2 (1964), 367-377.

10. W. S. Massey, On the cohomology ring of a sphere bundle, J. Math. Mech. 7 (1958), 265-290.

11. W. S. Massey and F. P. Peterson, On the cohomology algebra of fibre bundles whose fibre is totally nonhomologous to zero, Mimeographed notes, Univ. of Washington, Seattle, 1963.

12. - The cohomology structure of certain fibre spaces. I, Topology 4 (1965), 47-65.

13. J.-P. Meyer, Functional cohomology operations and relations, Amer. J. Math. 87 (1965), 649-683.

14. R. E. Mosher, Functional cohomology operations and secondary characteristic classes,

Ph.D. Thesis, Massachusetts Institute of Technology, Cambridge, Mass., 1962.

15. F. P. Peterson, Functional cohomology operations, Trans. Amer. Math. Soc. 86 (1957), 187-197.

16. F. P. Peterson and N. Stein, Secondary characteristic classes, Ann. of Math. (2) 76 (1962), 510-523.

17. N. E. Steenrod, Cohomology invariants of mappings, Ann. of Math. (2) 50 (1949), 954-988.

18. N. E. Steenrod and D. B. A. Epstein, Cohomology operations, Annals of Mathematics Studies, No. 50, Princeton Univ. Press, Princeton, N. J., 1962.

19. N. E. Steenrod and J. H. C. Whitehead, Vector fields on the n-sphere, Proc. Nat. Acad. Sci. U.S.A. 37 (1951), 58-63.

\section{California State College, Long BeACH, CALIFornia}

